Case Report

\title{
Brucellosis Spondylodiscitis: A case report
}

\author{
$\underline{\text { Nasrin Milani }^{1}}{ }^{(D)}$, Reza Ahmadi $^{2}$
}

\begin{abstract}
Brucellosis is an endemic disease in Iran that is transmitted from animal to human. The clinical manifestations of brucellosis are nonspecific and its complications involve bones and joints. Brucellosis osteomyelitis can mimic the manifestations of neoplasms; therefore, it cannot readily be diagnosed in some cases. Under such circumstances, high incidence can be a helpful factor.

We reported a 49-year-old man patient who presented with chronic musculoskeletal pain in the lower back. Initially, he was treated for osteoarthritis due to several negative serological tests for brucellosis. He was referred to a specialized clinic with no recovery achievement after 5 months treatment. The musculoskeletal pain together with the patient's occupation (animal husbandry), was resulted in a high clinical suspicion of brucellosis. Consequently, the patient underwent another serological test and Magnetic resonance imaging (MRI). He was diagnosed with brucellosis-induced spondylodiscitis by positive serology result and bone involvement on MRI. Regular treatment was then started, which led to clinical and radiological improvement at the end.

With regard to chronic symptoms of the disease and despite the negative results of serological tests, it is recommended that radiological diagnostic methods such as MRI should be adopted in cases where there is a clinical suspicion of brucellosis.
\end{abstract}

Keywords: Brucellosis, Osteomyelitis, Spondylitis 27(3): 308-313. [Persian].

DOI http://doi.org/10.32592/JBirjandUnivMedSci.2020.27.3.109

Received: March 27, 2020

Accepted: May 19, 2020

\footnotetext{
${ }^{1}$ Department of Internal Medicine, Mashhad University of Medical Sciences, Mashhad, Iran.

${ }^{2}$ Department of Family Medicine, Mashhad University of Medical Sciences, Mashhad, Iran
} $r+\Lambda$

Corresponding author; Department of Family Medicine, Mashhad University of Medical Sciences, Mashhad, Iran. Tel: +985137684843Ｆax: $985137684843 \quad$ Email: Milanin@mums.ac.ir/ nasrinmilani@gmail.com 


\title{
اسبونديلوديسكيت بروسلايى: معرفى يك مورد
}

\author{
نسرين ميلاني 'D') رضا احمدى')
}

\section{جكيده}

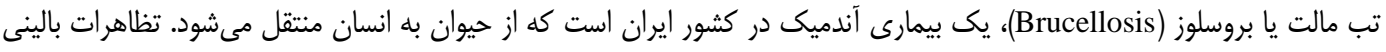

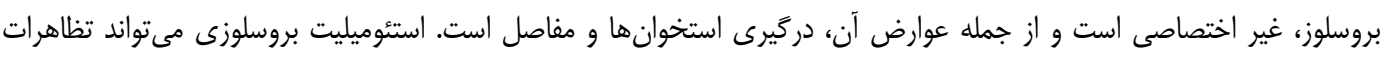

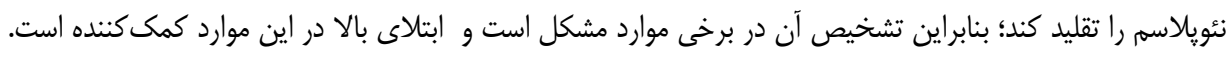

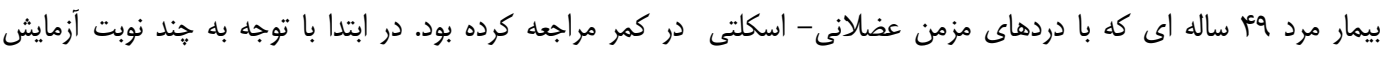

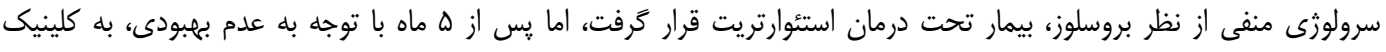

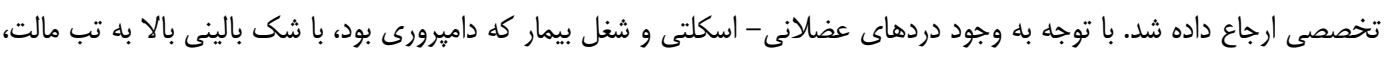

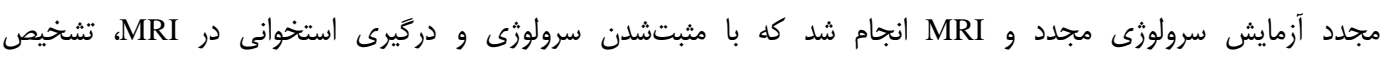

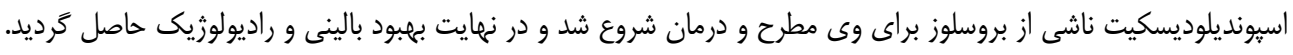

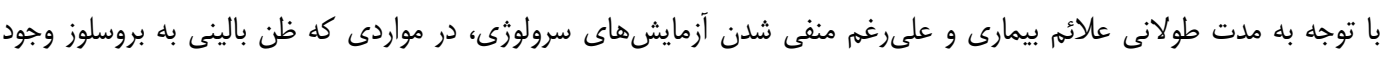

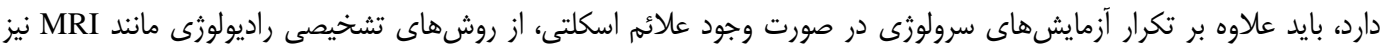

استفاده شود.

وازههاى كليدى: بروسلوز، استئوميليت، اسبونديليت

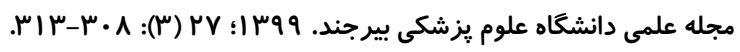

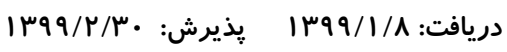

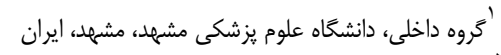

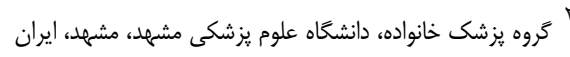

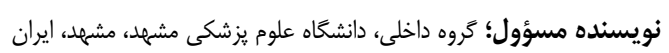

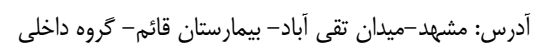
nasrinmilani@gmail.com /Milanin@mums.ac.ir بست الكترونيكى

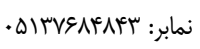

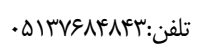


بروسلايى گزارش شد در ابتلا با تشخيص سرطان متاستاتيك

مقدمه

تحت راديوترايى قرار گرفته بود (ع).

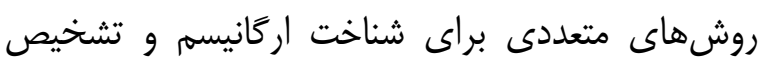

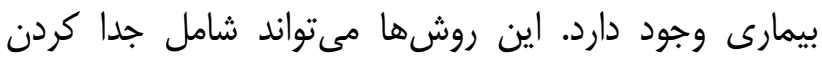

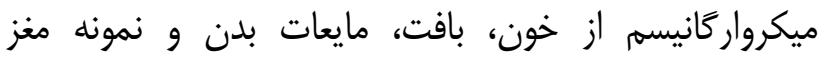

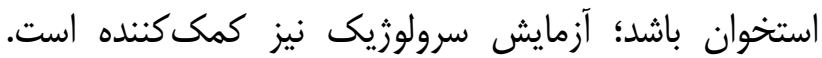

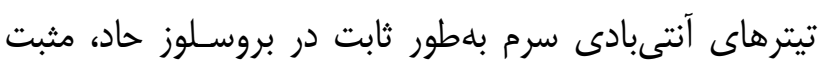

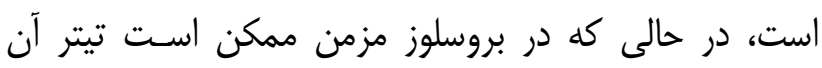
هايين و يا منفى باشد كه در اين شرايط جداسـازى بـاكترى از

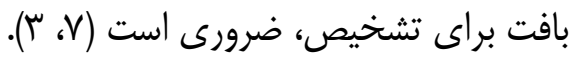

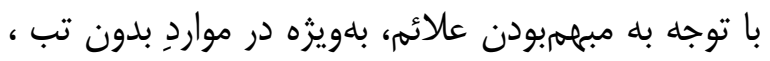

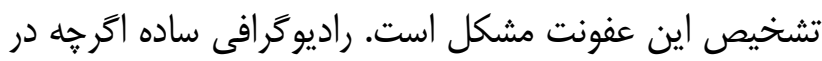

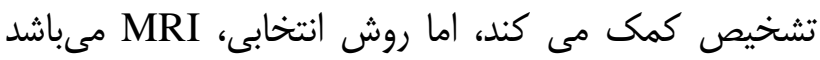

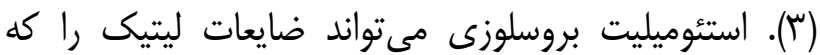
تقليدكننده نئويلاسم است، ايجاد كند. تشخيص ائن اين موارد در

صورت نداشتن شك بالينى بالا بسيار مشكل است (ّ). بهعلت اهميت تشخيص سريع استئوميليت مهرهها

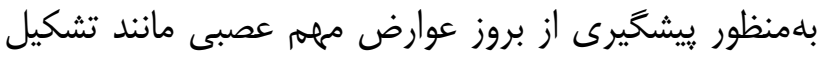

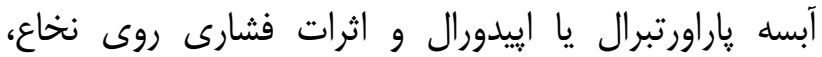
آشنايى يزشكان با بروسلوز اهميت بالايى دارد.

شرح مورد

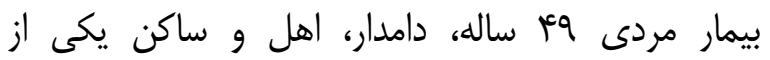

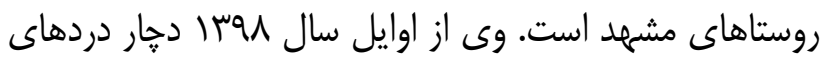

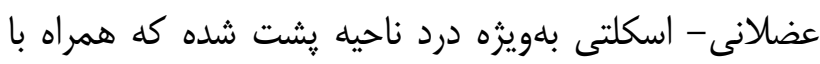
تعريق مختصر، ضعف و بىاشتهايى بوده است. بيمار קند بار بهار

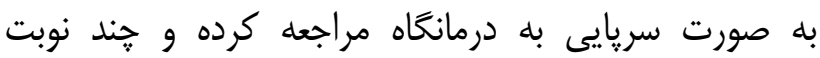
آزمايش سرولوزى بروسلوز منفى داشته است. در نهايت تحت درانه

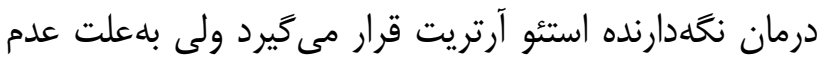

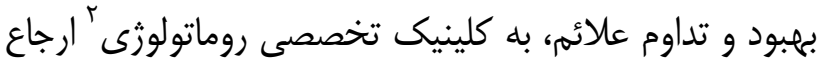
مىشود.
تب مالت يا بروسلوز (Brucellosis)، بيمارى باكتريايى

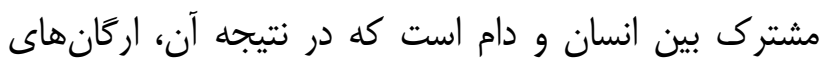
مختلف بدن درگير شده و باعث طيف وسيعى از تظاهرات

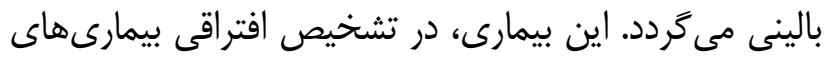

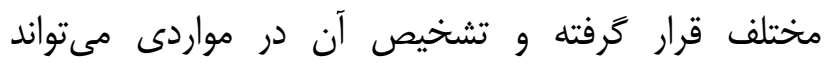

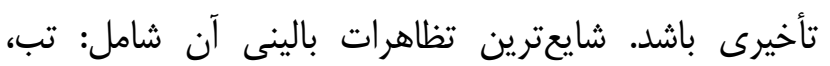
تعريق، لرز، خستخى، سردرد و دردهاى عضلانى - اسكلتى و

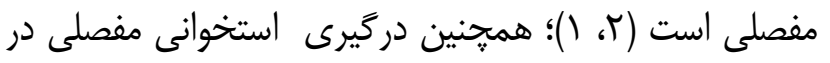

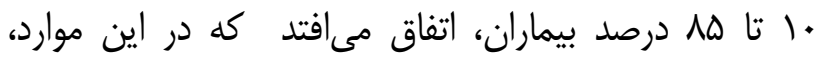

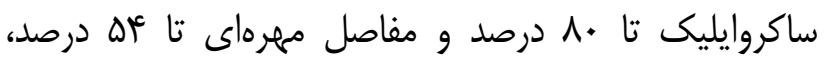

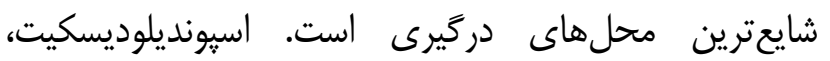

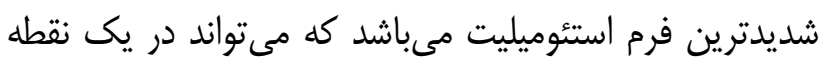
از ستون فقرات و يا در مناطق جدا از هم بروز نمايد (1)

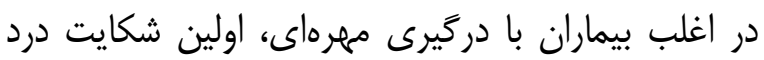
لوكاليزه و تندرنس 'در ستون مهرههاست. در كمتر از نيمى از

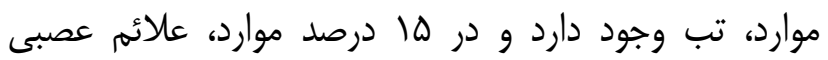
بهعلت فشار به نخاع ديده مى شود (بّ). آبسه إِيدورال،

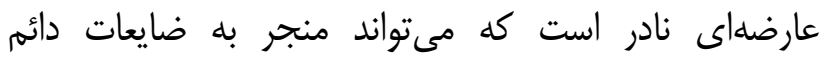

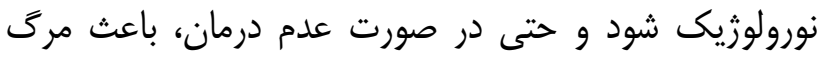
كردد (1) (1)

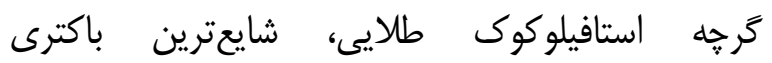
ايجادكننده عفونت اسبونديلوديسكيت است اما در نواحى إسى

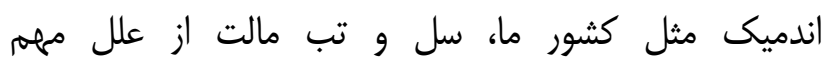
استئوميليت مهرهاى هستند. مطالعاتى در اين زمينه در ايران

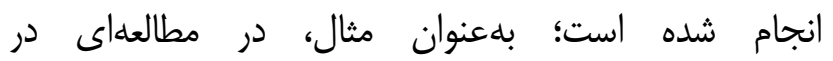

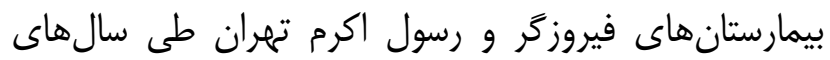

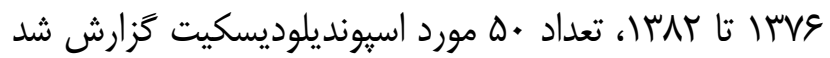

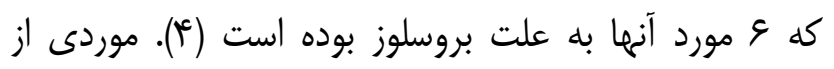

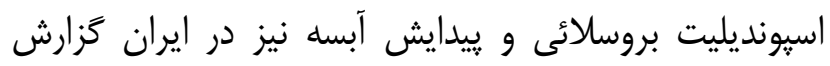

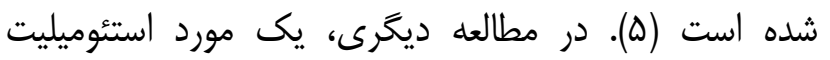

\footnotetext{
${ }^{1}$ Tenderness
} 
آزمايش شد كه در نهايت با فاصله •م روز از آزمايش نوبت اول در نهايت آزمايش سرولوزى بروسلوز، مثبت كرديد.

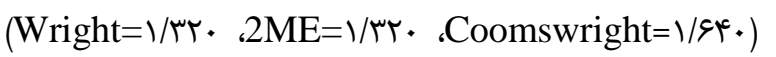
بلمنظور بررسى بيشتر، MRI لومبوساكرال براى بيمار انجام

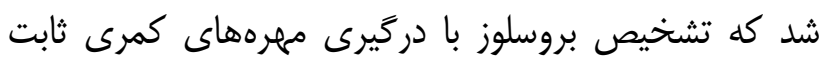
كرديد.

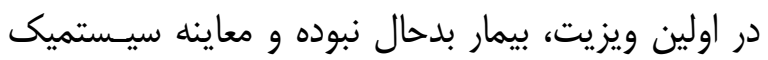

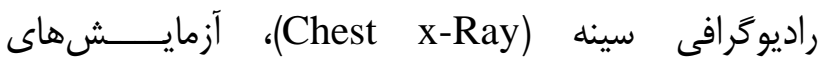
بيوشـيمى در حـد طبيعى بود. در بررسى تكميلى، سديمان

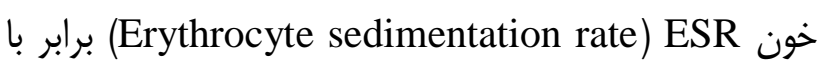

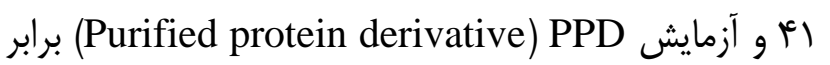
ᄉ بود. با توجه به شك بالينى به بروسلوز و پايدار ماندن علائم

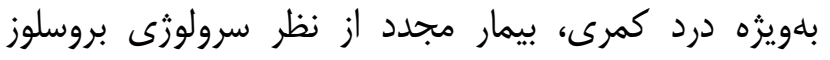

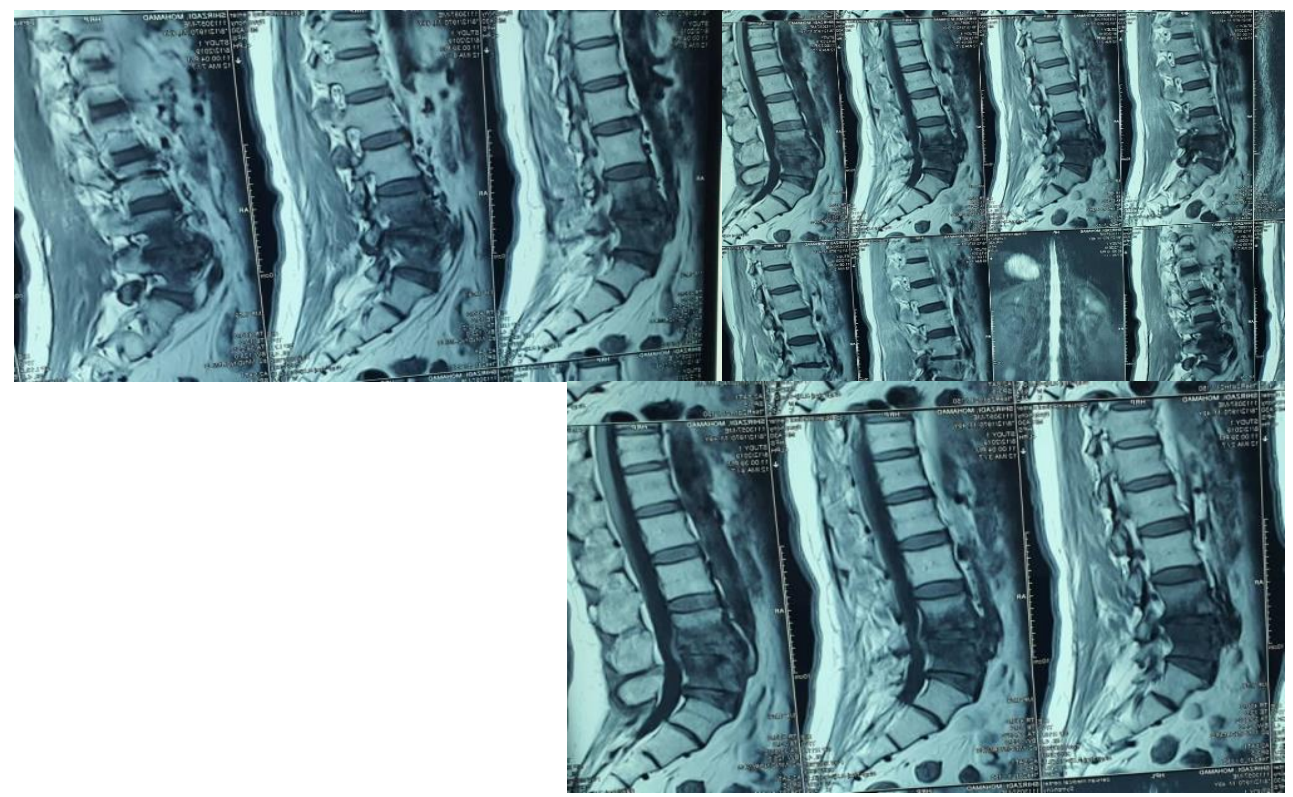

شكل ا- تصاوير مقطع سازيتال و ياراسازيتال لومبوساكرال مربوط به بيش از درمان

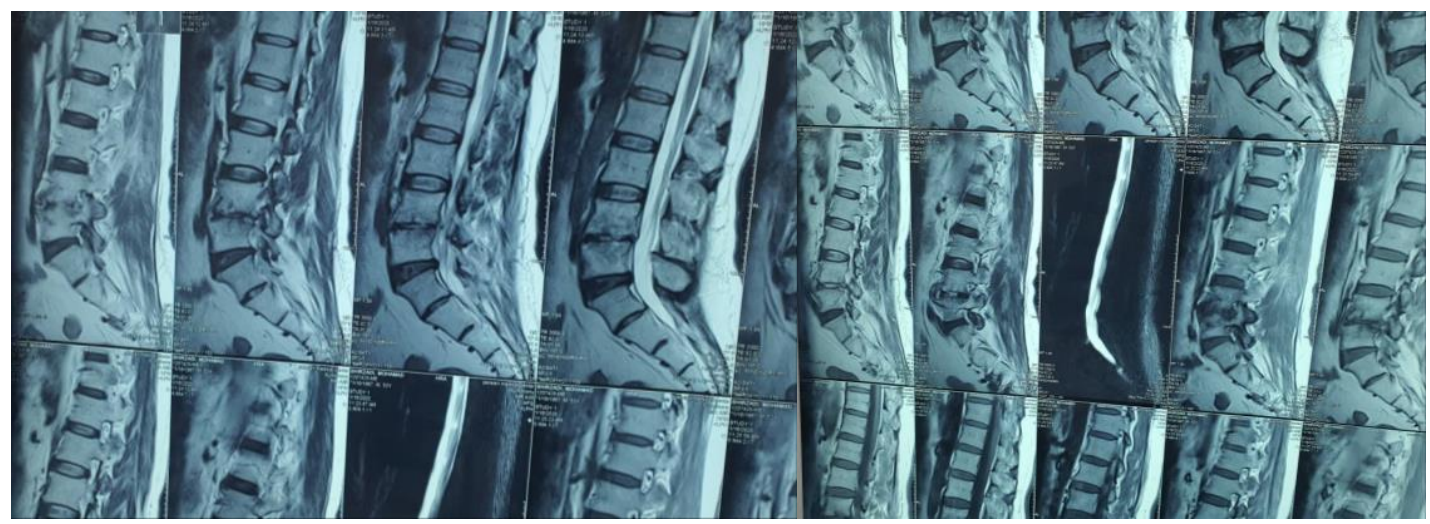

شكل ؟ - تصاوير مقطع سازيتال و پار اسازيتال لومبوساكرال مربوط به بعد از درمان

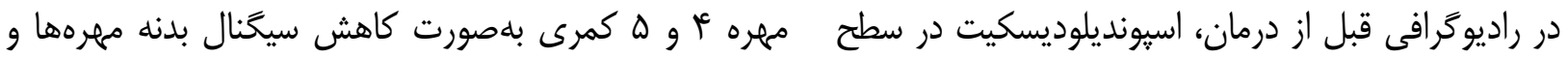




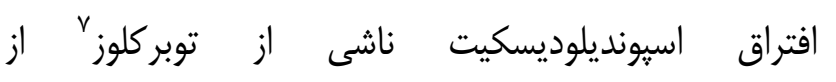

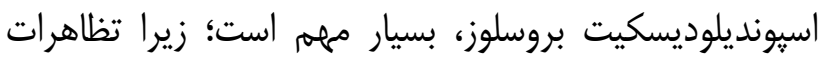

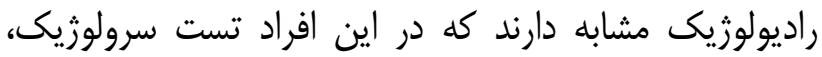

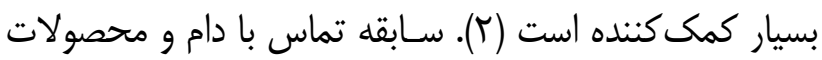

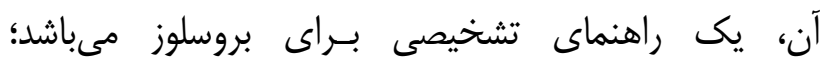

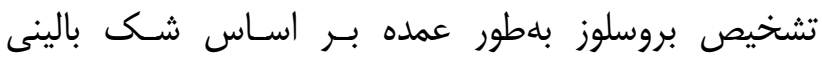

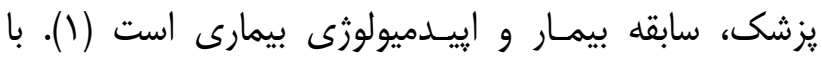

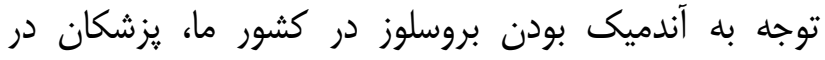

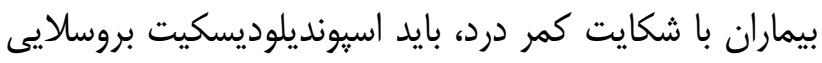
را بلعنوان يكى از تشخيص افتراقىها مدّ نظر داشته باشند؛ با بان

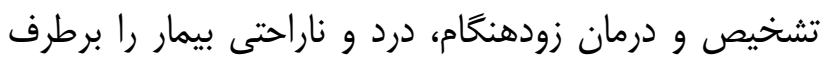

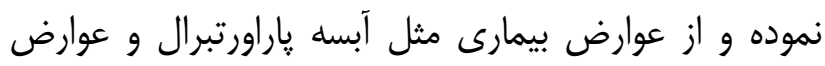

$$
\text { عصبى ييشگيرى كرد. }
$$

از جمله روشهاى تشخيصى انتخابى دركيرى ستون

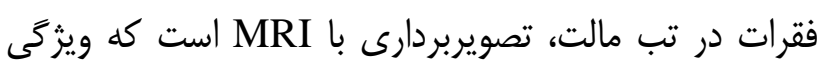
يإيين براى تشخيص قطعى ضايعات استئوآرتريكولار دارد؛ اما

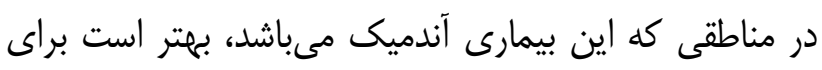

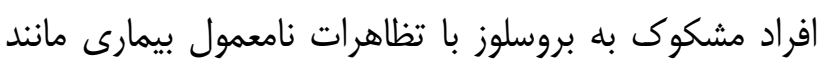

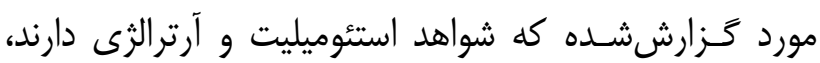

MRI

\section{تقدير و تشكر}

نويسندكان، از خانم دكتر سمانه خواجه نصيرى بابت بابت همكارى در مطالعه تقدير و تشكر مى كنند.

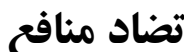

نويسندًان تعارض منافع با مطالب درجشه در مقاله ندارند.

\footnotetext{
${ }^{6}$ Spondylitis

${ }^{7}$ Tuberculosis
}

ديسك بين مهرهاى همراه با كاهش ارتفاع ديسك و نامنظمى صفحه انتهايى (End plate) مهرهماى مبرى مجاور

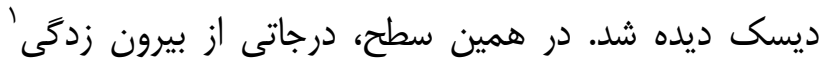

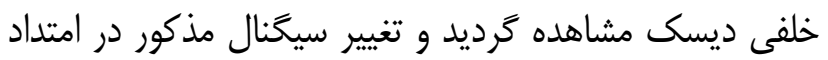

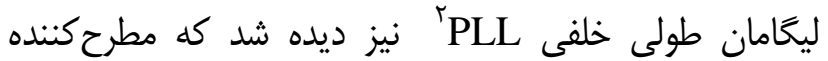
التهاب يا تجمع اكزوداى التهابى در فضاى مذائ دذكور قدام

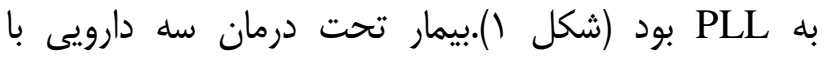

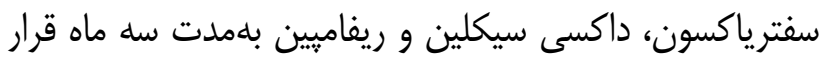

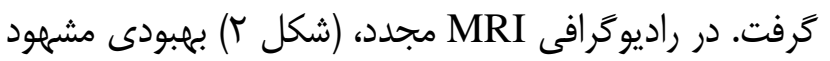
بود؛ بهكونهاى كه در تصاوير بعد از انجام دردام درمان، تغيير

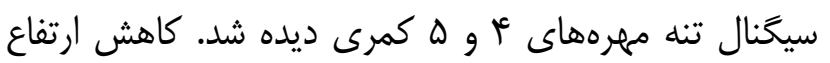
ديسك بينمهرهاى ديده شد، ولى التهاب و اتزوداى التهابى

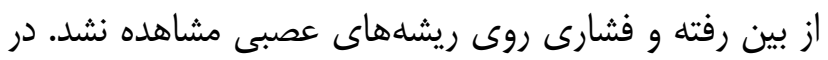

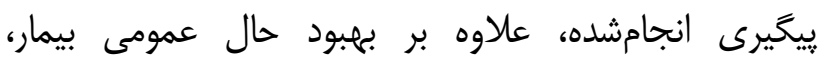

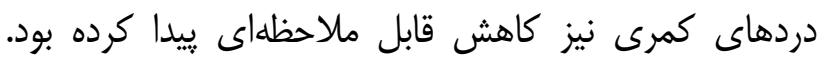

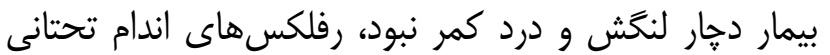
وى نرمال بود و اختلال حس و حركت مشهود نداشت (شكل

لازم به ذكر است تمام اطلاعات با توجه به رضايتنامه

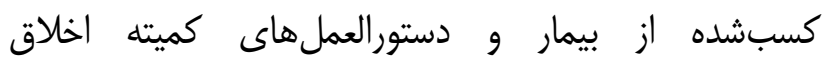
IR.MUMS.REC.1399.374 در مقاله ذكر شده است.

\section{نتيجه كيرى}

سيسته اسكلتى شايع ترين ابتلا را در بيمارى بروسلوز

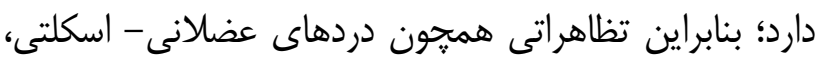
استوآرتريكولار (به عنوان شايعترين تظاهر)، ساكروايليت و وائن

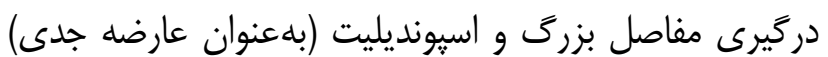

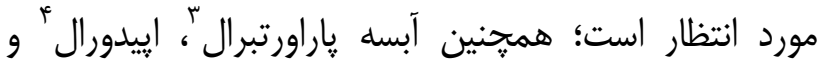

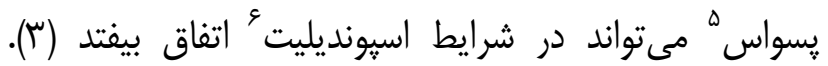

${ }^{1}$ Protrusion

${ }^{2}$ Posterior longitudinal ligament

${ }^{3}$ Paravertebral

${ }^{4}$ Epidural

${ }^{5}$ Psoas 
1- Esmaeilnejad-Ganji SM, Esmaeilnejad-Ganji SMR. Osteoarticular manifestations of human brucellosis: A review. World J Orthop. 2019; 10(2): 54-62. doi: 10.5312/wjo.v10.i2.54.

2- Pourbagher A, Pourbagher MA, Savas L, Turunc T, Demiroglu YZ, Erol I, et al. Epidemiologic, clinical, and imaging findings in brucellosis patients with osteoarticular involvement. AJR Am J Roentgenol. 2006; 187(4): 87380. doi: 10.2214/AJR.05.1088.

3- Bennett JE, Dolin R, Blaser MJ. Mandell, Douglas, and Bennett's Principles and Practice of Infectious Diseases. $8^{\text {th }}$ ed. Elsevier; 2014.

4- Talebi Taher M, Mirzaei A. A Survey of 50 Cases of Spondylodiscitis: Clinical, Microbiological, Radiological Features and Treatment. RJMS. 2007; 14(55): 123-31. [Persian]

5- Vaziri S, Soleyman Meigouni S, Janbakhsh AR, Mansouri FA, Sayyad B, Afsharian M. Brucella spondylitis and paraspinal abscess (Case Report). J Gorgan Univ Med Sci. 2008; 10(3): 87-90. [Persian]

6- Ramezani Awal RiabiH, Ahmadi R. A Rare Osteoarticular Brucellosis in Gonabad City, Iran: A Case Report. Razavi Int J Med. 2015; 3(2): 1-3. doi: 10.5812/rijm.21183

7- Al Dahouk S, Tomaso H, Nockler K, Neubauer H, Frangoulidis D. Laboratory-based diagnosis of brucellosis--a review of the literature. Part II: serological tests for brucellosis. Clin Lab. 2003; 49(11-12): 577-89. 